\title{
Biology: In the Language of the People
}

\author{
Tim Marler and Dona Odell* \\ Department of Biology, USA
}

*Corresponding author: Tim Marler and Dona Odell, Department of Biology, USA.

\author{
Received Date: January 19, 2019 \\ Published Date: February 07, 2019
}

\section{Introduction}

As technology and the ability to gather ever-growing amounts of data move further into the realms of biology and human performance, communication and transparency become increasingly important. As scientists, engineers, and medical practitioners, we can often lose sight of the idea that this communication should extend to the non-technical public. In the words of William Butler Yeats, "Think like a wise man but communicate in the language of the people." We would all do well to remember such advice even during scientific pursuits and documentation.

The headlines are cluttered with stories about technological advancements in robotics, autonomous systems, artificial intelligence, and other fields. These fields often depend on large sets of data or "big data" ${ }^{1}$ " from which mathematical models are formed and used to predict behavior or govern actions of machines. We are moving from a purely machine-based society to a data - or information - based society.

Increasingly, the ability to gather this data extends not only to machines and systems, but also to groups of people and, more importantly, to the individual. To be sure, biostatistics and biometrics have always involved complex biological data not easily digestible by non -scientists. But now, data is accumulated in unprecedented amounts and is increasingly personal. American companies spent over $\$ 19$ billion in 2018 acquiring and analyzing consumer data $^{2}$, and policies for handling that data have not kept pace. This technological evolution can have significant personal impacts on wide swaths of the public. A cell phone or an automotive GPS system may track a user's location. A web page may track someone's interests. A digital bracelet may store one's physical habits, and dieting software may store a user's eating habits on a server. Even an individual's fundamental biological "code," someone's DNA, can be mapped, stored, and used for predictions. There is a shift from just tracking where we are and what we do, to who we are and how we each function. Consequently, clear communication becomes all the more important.

While these data-gathering capabilities can yield significant good, they can also present challenging ethical and policy issues. Indeed, there is public discussion about privacy and scientific boundaries, but these discussions hinge on an informed public. While the convergence of data-gathering technology that centers on human performance continues to warrant sound scientific practice and careful experimentation, keeping the public informed should not be overlooked. Of course, practitioners and scientists and engineers must discuss among themselves new capabilities and results, but it is increasingly important for technicians to convey their methods and results to the public. A key tool in developing appropriate and ethical policies is transparency. In the case of biometrics, this now applies not only to the developers and the users but to the sources of data, which can be the general public. Neglecting this obligation risks public misunderstanding, or even disengagement and a consequent reduction in data ${ }^{3}$.

Politicians and the modern media have no monopoly on the idea of so-called fake news, or truth decay ${ }^{4}$. Although science and engineering have not traditionally been known for spinning results, they are by no means immune. There is now an opportunity to be proactive as practitioners in the fields of biostatistics and biometrics in order to help foster transparency and understanding with the general public.

${ }^{1}$ https://www.forbes.com/sites/gilpress/2014/09/03/12-big-data-definitions-whats-yours/\#2d7b93d713ae

${ }^{2}$ https://www.wired.com/story/2018-power-of-personal-data/

${ }^{3}$ https://www.rand.org/blog/2018/12/data-breaches-could-cause-users-to-opt-out-of-sharing.html

${ }^{4}$ https://www.rand.org/pubs/research_reports/RR2314.html 
Technicians have a responsibility to inform non-technical readers about topics of the day, like genetics, sensors, big data, biology, and so forth. In some respects, such communication between the scientific community and the general public can be more challenging than intra-scientific discussions. Communicating information from a body of research concerning biostatistics and biometrics to a broad and diverse audience requires an understanding of what the audience's perspective is and then enhancing that perspective. It may be necessary to understand the culture of each audience and to consider the best tools and the best language to promote understanding and acceptance of the work. Authors should consider whether they are using the words, examples, and models that connect with a broad audience most effectively.

Presenting complex material to both technical and nontechnical audiences may require two different approaches and two different publications. There is certainly no need to sacrifice scientific precision for ease of understanding but presenting results in a way that can only be understood by fellow practitioners is no longer sufficient. There is a need to focus on clarity and communicate with the broadest possible audience. In the current discussion of vaccinations, for example, it is important to convey clearly to the general public the data that show how vaccinations have prevented the deaths of millions, not only in foreign lands but in this country as well. To communicate this to the broadest audience, social media and pictorials, for example, may provide a better outlet than an esoteric journal.

As technology and related data-acquisition accelerate and link directly to individuals, it is no longer sufficient merely to form a strong hypothesis and test it in the context of a rigorous scientific method. It is no longer sufficient to gauge success by scientific results. Rather, it is now the scientist's responsibility to consider the source of the data, and to communicate results to the public in the language of the people.

Tim Marler is a research engineer at the nonprofit, nonpartisan RAND Corporation and a professor at the Pardee RAND Graduate School. Dona Odell is freelance science writer after having taught biology for 50 years.

\section{Acknowledgement}

None.

\section{Conflict of Interest}

No conflict of interest. 